\title{
Two Types of Quantificational Modalized Genericity, and the Interpretation of Bare Plural and Indefinite Singular NPs
}

\author{
Yael Greenberg \\ Bar Ilan university.
}

The main goal of this paper is to develop a formal analysis which accounts for both the strong similarities, as well as a number of unexpected differences between two types of generic sentences in English, which minimally contrast in the type of subject: those with indefinite singular subjects, as in (1) (IS sentences, henceforth), and those with bare plural subjects, as in (2) (BP sentences, henceforth):

(1) A grizzly bear snores very loudly (- An IS sentence)

(2) Grizzly bears snore very loudly (- A BP sentence)

I start (in section 1) by reviewing the uniform quantificational-modal representation traditionally assigned to minimal pairs like (1) and (2), and its advantages. In section 2 I present the problem for the uniform representation, namely a number of old, as well as newly observed differences between such minimal pairs. Section 3 deals with several attempts in the literature to characterize and explain these differences, and their shortcomings. My alternative account of both the similarities and differences between the two types of sentences is to take them, on the one hand, to have the same basic semantic structure (headed by a modalized generic quantifier, Gen), but to differ in the type of modality, i.e. the type of accessibility relation restricting Gen. The intuition behind this alternative, as well as the formal analysis are presented in section 4 . In section 5 I show how the formal analysis accounts for the full range of similarities and differences between IS and BP sentences, presented above. Finally in section 6 I suggest a preliminary direction for deriving the semantic difference between the two types of sentences (in terms of their availability with the two distinct accessibility relations) from the distinct semantics of the IS and BP noun phrases subjects in these sentences.

\section{The uniform representation and its advantages}

Minimally contrasting IS and BP sentences, like (1) and (2) above, look very very similar, in fact - almost synonymous. Indeed, almost all theories of genericity analyze them the same, as equally expressing nonaccidental genericity (I-genericity, in Krifka \& Gerstner's 1987 terms, or Characterizing Genericity, in Krifka et al's 1995 terms), i.e. as having some version or other of the tripartite structure in (3), suggested By Chierchia 1995 (where Gen is a nonovert unselective quantifier binding the individual $(\mathrm{x})$, and situation (s) variables introduced by the subject predicate and the VP predicate respectively, and $\mathrm{C}$ is a variable yielding contextually relevant situations involving individuals) ${ }^{1}$ :

(3) Gen, $\mathrm{x}, \mathrm{s}$ [grizzly bear $(\mathrm{x}) \wedge \mathrm{C}(\mathrm{s}, \mathrm{x})$ ] $\rightarrow$ [snore loudly $(\mathrm{s}, \mathrm{x})$ ]

Paraphrase: "It generally holds for individual grizzly bears and contextually relevant (e.g. sleeping) situations involving them, that such individuals snore loudly in such situations" 
As for the semantics of Gen, it is generally assumed (in e.g. Dahl 1975, Wilkinson 1991, Chierchia 1995, Krifka 1995) that it is universal and modalized, i.e. that it universally quantifies not only over individuals and situations, but also over possible worlds, restricted by some sort of modal base (in Kratzer's 1981 terms), or accessibility relation. Since I deal a lot with this accessibility relation below, I wrote (3) explicitly as in (4) :

$$
\begin{aligned}
& \forall \mathrm{w}^{\prime}\left[\mathrm{w}^{\prime} \text { is appropriately accessible from } \mathrm{w}\right] \rightarrow \\
& \left.\left.\quad\left[\forall \mathrm{x}, \mathrm{s} \text { [grizzly bear }\left(\mathrm{x}, \mathrm{w}^{\prime}\right) \wedge \mathrm{C}\left(\mathrm{s}, \mathrm{x}, \mathrm{w}^{\prime}\right)\right] \rightarrow \text { [snore loudly }\left(\mathrm{s}, \mathrm{x}, \mathrm{w}^{\prime}\right)\right]\right]
\end{aligned}
$$

Paraphrase :"In all worlds $w$ ' appropriately accessible from the world of evaluation, w, every grizzly bear, in any contextually relevant situation (e.g. every sleeping situation) is snaring very loudly".

Following Kratzer's 1981 analysis of modal verbs, Krifka et al 1995 suggest that the basic accessibility relation of the generic quantifier in (4) is similar to that of modal verbs like must, i.e. that it is supplied by context and can vary between epistemic, deontic, stereotypical, etc.

The uniform representation in (4) indeed successfully captures some important similarities between minimally contrasting IS and BP sentences. Specifically, the quantification over individuals and situations captures the fact that both types of sentences are felt to express generalizations over such entities ${ }^{2}$. The fact that the universal statement in (4) (over individuals and situations) is under the scope of a modal operator, i.e. it is required to hold in a set of accessible worlds, rather than merely in the actual world, captures the intuition that the generalizations expressed by both IS and BP sentences like (1) and (2) are taken to be "law-like", or "nonaccidental". Among other things, this is indicated by the fact that both (1) and (2) above support the counterfactual statement in (5):

If this were a grizzly bear it would probably snore very loudly as well.

The variability of the accessibility relation, i.e. the fact that in each case the universal statement is required to hold in a different set of worlds, successfully captures the fact that both IS and BP sentences can express generalizations of different "flavors", e.g. epistemic ones as in (1) and (2) above, and as in (6), normative, or deontic ones, as in (7), definitions, or linguistic ones, as in (8), etc.:

(6) Cats have four legs / A cat has four legs

(7) Dogs owners pay tax on them / A dog owner pays tax on it

(8) Bachelors are never married men / A bachelor is never married man

In (6), for example, the universal statement (in this case "every dog has four legs in all situations") holds in all worlds which are epistemically accessible from the actual world w, i.e. it follows from what is known in w. In (7) it holds in all worlds which are deontically accessible, i.e. it follows form what is commanded in w. And in (8) it holds in all worlds which are linguistically accessible from w, i.e. where the language is interpreted as in $\mathrm{w}$. 


\section{The problem for the uniform representation: differences between minimally} contrasting IS and BP sentences.

However, despite the success of the uniform representation to account for the similarities between minimally contrasting IS and BP sentences, it cannot account for a number of differences between them.

The observations about some of these differences are, in fact, rather old. Burton Roberts 1977, for example, observed that minimal pairs like (1) and (2), or like (9) and (10) are not really synonymous. IS sentences express a high level of law-likeness: "definitional" or analytic statements, so (9), for example, can be paraphrased as "To be a table is to have four legs". In contrast, BP sentences, like (10) can also express a lower level of, "law likeness", "descriptive" or "inductive" generalizations, about patterns (rather than rules)), concerning, e.g. the number of legs that tables generally have:

(9) A table has four legs

(10) Tables have four legs

Moreover, the felicity of IS sentences (under the generic interpretation), appears to be much more restricted than that of their BP counterparts. For example, Lawler 1973, Burton Roberts 1977 and others observed that IS sentences as in (11a)-(11d) are odd, or infelicitous as generic, whereas the BP sentences in (12a)-(12d) are perfectly felicitous under this reading (although probably false):
a. \#A madrigal is popular
b. \# A room is square
c. \# An uncle is garnlous
d. \# A man is blond
a. Madrigals are popular
b. Rooms are square
c. Uncles are gamulous
d. Men are blond

More differences can be seen with IS and BP sentences whose subject express what I intuitively call "extremely unnatural classes", as in (13) and (14), respectively:

(13) a. A Norwegian student whose name ends with 's' or 'g' wears thick green socks. (Salient existential reading of the subject)

b. An American linguist born to a famous singer in June 1954 writes very technical papers (Salient existential reading of the subject)

(14) a. Norwegian students whose name ends with ' $s$ ' or ' $g$ ' wear thick green socks. (Salient generic reading of the subject)

b. American linguists born in June 1954 to famous singers write very technical papers (Salient generic reading of the subject)

As can be seen in (13), with IS sentences such "extremely unnatural" subjects get a salient existential interpretation (in fact they are interpreted as wide scope existentials, e.g. about specific Norwegian students), and the sentences themselves are odd as generic. In contrast, with such BP sentences, as in (14), the subjects are freely 
interpreted as generic, (e.g. (14a) is perfectly fine as a generalization about Norwegian students whose names end with ' $s$ ' or ' $g$ ').

A similar pattern can be seen in "temporally restricted generics" (Greenberg 1998,2002 ). Unlike the IS sentences in (15), which are infelicitous under the generic reading of the subject, the minimally contrasting BP counterparts in (16) are perfectly fine ${ }^{3}$ :

a. \# An Italian restaurant is closed tonight

b. \# An accountant is very busy this week

a. Italian restaurants are closed tonight

b. Accountants are very busy this week

It is clear that the uniform representation in (4) above, cannot handle these differences between IS and BP sentences. In order to account for the data, there are two basic questions we need to answer: (A) How can we characterize these differences ? (B) How can we explain them, i.e. derive them from independently motivated differences between IS and BP noun phrases?

The answer to neither question is trivial or easy. To see that, let me look first at some traditional attempts to account for the data.

\section{Previous attempts to characterize and explain the differences between IS and BP sentences, and their shortcommings}

Based on data as in (11) and (12) above, Lawler 1973 suggested that IS sentences are felicitous only when their VP express properties which are "essential" or "inherent" of the subjects, whereas BP ones have no such restriction - they can freely combine with purely "accidental", noninherent properties. According to Lawler, then, (11a) above, repeated here as $(17 \mathrm{a})$, is infelicitous as generic since being popular is an accidental, noninherent property of madrigals, whereas (17b) is fine since being polyphonic is essential / inherent of madrigals:
a. \#A madrigal is popular
b. A madrigal is polyphonic

This intuitive characterization is quoted in a number of generic theories. However, as shown in Cohen 20011999 and Greenebrg 2002, the main problem with it is that there are, in fact, many perfectly felicitous IS sentences which combine with clearly accidental nonessential, noninherent properties, like (18a-b), or which, moreover, combine with clearly false properties of their subjects (like 19a-b):
a. A carpenter earns very little.
b. A refrigerator costs $\$ 1000$
a. A dog has three legs
b. A pine tree has wide leave

(18b), for example, is perfectly fine as generic although costing $\$ 1000$ is not considered an essential property of refrigerators. Moreover, one clearly wouldn't want to claim that having three legs is an essential property of dogs, or that having wide 
leaves is an essential property of pine trees, but nonetheless, $(19 a, b)$ are perfectly felicitous as generic.

What these problems suggest is that the essential / nonessential distinction is not the right way to "slice the cake" correctly, i.e. to distinguish between felicitous IS sentences (e.g. the clearly false (19a)), and infelicitous ones (like (11d)). Before presenting an alternative characterization, however, let us look at an attempt to explain these differences, namely Krifka et al's 1995 suggestion to assign minimally contrasting IS and BP sentences two completely different semantic structures ${ }^{4}$.

Specifically, the claim is that IS sentences are necessarily characterizing (I-) generics, i.e. must have a modalized generic quantifier, of the type described in section 1 above, and that the modal nature of this quantifier somehow leads to the "analyticity" and "essentiality" restrictions on them. In contrast, all BP sentences can have a nonquantificational, nonmodal Direct Kind Predication structure, and the abseence of modal operator enables them to be interpreted as generics even when combining with "nonessential" / "accidental" properties.

Now, Krifka \& Gerstner 1987 independently showed that there are indeed BP sentences which are interpreted as generic, although they lack a modalized generic quantifier. E.g. (20a) clearly does not express a nonaccidental generalization over individual Dodos, and hence should not be represented as in (20b). Instead, it is usually represented as in (20c), where the VP property is directly predicated of the kind, denoted by the BP NP "dodos" (represented with capital letters), i.e. as a Direct Kind Predication Structure. The ungrammaticality of (20d) shows that unlike BP sentences IS ones cannot be interpreted as such Direct Kind Predication structures (presumably since, unlike BP NPs, IS ones cannot denote kinds):
a. Dodos became extinct in 1713
b. * Gen $\mathrm{x}, \mathrm{s}[\operatorname{Dodos}(\mathrm{x}) \wedge \mathrm{C}(\mathrm{s}, \mathrm{x})] \rightarrow$ [became extinct in $1713(\mathrm{~s}, \mathrm{x})]$
c. became extinct in 1713 (DODOS)
d. * A dodo became extinct in 1713

These assumptions are by now standard. The innovation in Krifka et al's suggestion is in applying this nonmodalized, nonquantificational Direct Kind Predication structure not only to BP sentences like (20a) but also to e.g. (21a), and presumably also to (22a) and (23a):
a. Uncles are garrulous
b. garrulous (UNCLES)
a. Men are blond
b. Blond (MEN)
a. Italian restaurants are closed tonight
b. closed tonight (ITALIAN RESTAURANTS)

There are two basic problems with Krifka et al's approach. Firstly, the mere presence of a modalized generic quantifier in the semantics of IS sentences, with the traditional epistemic, deontic, stereotypical etc. accessibility relations used by Krifka et al, is simply not enough to explain the restrictions on their felicity. Specifically, the presence of such a modalized quantifier wrongly predicts IS sentences like (24a) to have exactly the same status as e.g. (24b):
a. \# A man is blond 
b. A dog has three legs

The reason is that neither universal statements in (25a) and (25b) (corresponding to (24a) and (24b), respectively), follow from what is known, commanded, stereotyped etc. in $\mathrm{w}$, i.e. neither of them holds in all worlds which are epistemically, deontically, stereotypically, etc. from the actual world w:
a. Every man is blond
b. Every dogs has three legs

Thus, while Lewler's essential / nonessential distinction wrongly predicts both (24a) and (24b) to be equally infelicitous (since in both the VP expresses a nonessential property of the subject), Krifka et al's suggestion in fact wrongly predict both IS sentences to be equally felicitous (and false). As I will show below, a more sophisticated accessibility relation has to be used in order to "slice the cake" correctly i.e. to distinguish between felicitous and infelicitous IS sentences.

The second problem has to do with Krifka et al's analysis of BP sentences like (21)-(23) above. In section (1) we saw that the uniform representation (in e.g. ((4)) above accounts nicely for the similarities between minimally contrasting IS and BP sentences, but cannot handle the differences between them. Here we get the opposite situation: assigning such minimal pairs two completely different semantic structures attempts to account for the differences between them, but at the same time completely ignores the strong similarities between them. Specifically, assigning a nonquantificational, nonmodalized representation to BP sentences like (21a), (22a) and (24a) cannot account for the fact that, unlike e.g. Dodos are extinct, they are similar to IS sentences in expressing generalizations over individuals (individual uncles, men and Italian restaurants), and furthermore in supporting counterfactuals, e.g. (26), (27) and (28), respectively:

(26) If this were an uncle, it would be garrulous as well (pointing at a person who is not an uncle)

(27) If this were a man, he would be blond as well (pointing at a woman)

(28) If this were an Italian restaurant, it would be closed tonight as well (pointing at an Indian restaurant)

These facts suggest that, unlike Krifka et al's suggestion, the semantics of such BP sentences does contain a quantifier, which, moreover, has a modal nature.

\section{An alternative solution: Two types of nonaccidental genericity}

What we want, then, is to find a way to account for both the similarities and the differences between minimally contrasting IS and BP sentences. To do that I suggest that both types of sentences express nonaccidental genericity, but that they differ in the type of nonaccidentalness they express. More formally, that both types of sentences have the same basic, quantificational-modal, representation, of the sort we saw in section 1 above, but they differ in the type of modality - i.e. the type of accessibility relation available for them.

For a start, let's assume that both IS and BP sentences in (29a) and (29b) will have the same semantic structure as in (30). The difference between them will lie in 
the underlined part of the formula in (30), namely in the decision of which worlds ( $w^{\prime}$ ) will be considered appropriately accessible from $w$. The following sections specify how these two accessibility relations should be characterized:

a. A boy doesn't cry

b. Boys don't cry

$\forall \mathrm{w}^{\prime}$ [w' is appropriately accessible from $\left.\mathrm{w}\right] \rightarrow$

$\left[\forall \mathrm{x}, \mathrm{s}\left[\mathrm{boy}\left(\mathrm{x}, \mathrm{w}^{\prime}\right) \wedge \mathrm{C}\left(\mathrm{s}, \mathrm{x}, \mathrm{w}^{\prime}\right)\right] \rightarrow\left[\neg \operatorname{cry}\left(\mathrm{s}, \mathrm{x}, \mathrm{w}^{\prime}\right)\right]\right]$

Paraphrase: In all worlds appropriately accessible from $\mathrm{w}$, every boy, in all contextually relevant situations, doesn't cry.

\subsection{The intuitive difference between minimally contrasting IS and BP sentences}

Let me start by developing the intuition. The idea is that there are, in fact, two types of nonaccidental generalizations which are syntactically encoded in English ${ }^{5}$ through the distinction between IS and BP sentences. I call them "in virtue of" and "descriptive" generalizations ${ }^{6}$.

IS sentences only express "in virtue of" generalizations, asserting that the generalization is nonaccidentally true in virtue of some property, associated with the subject property, that the speaker has in mind, and the listener is supposed to accommodate. (31a), for example, can be intuitively paraphrased as in (31b):

a. A boy doesn't cry

b. The generalization "Every boy doesn't cry (in any relevant situation)" is nonaccidentally true in virtue of some property, associated with the property of being a boy (e.g. the property of being tough)

There are also IS sentences where the choice of the "in virtue of" property is not clear from the start. Hearing (32a), for example, one can accommodate any of the properties in (32b):

a. A woman in this place doesn't walk alone outside

b. In virtue of following the local religious or social norms...

In virtue of living in such a dangerous and violent place...

In virtue of being so beautiful and charming... (so every woman in this place is always accompanied by some man)

If uttered "out of the blue" it is not clear which "in virtue of" property the speaker has in mind, and the listener may end up accommodating the wrong "in virtue of" property, i.e. not the one the speaker had in mind. But the crucial assumption is that uttering such a sentence, the speaker really has in mind some "in virtue of" property which the listeners should accommodate to fully understand the sentence.

In contrast to IS sentences, BP ones are potentially ambiguous. The can express "in virtue of" generalizations, just like their IS counterparts, but also the second, "descriptive" type of generalizations, where they merely assert that "there is a pattern here", i.e. that the generalization is simply not accidental, not limited to the actual set of circumstances, but is expected to be true in other, nonactual circumstances as well. 
For example, although the BP (33a) can express an "in virtue of" generalization, just like there IS counterparts (paraphrased in (31a) above), on the basis of observing the behavior of many boys a speaker may also use it to express a descriptive generalization, paraphrased as in (33b):

a. Boys don't cry

b. The generalization "every boy doesn't cry (in all relevant, e.g. "tear inducing" situations)" is not accidental: not limited to actual boys in actual (relevant) situations, but is expected to hold for other, nonactual boys in other, nonactual (relevant) situations, as well."

Crucially, on the descriptive reading (33a) does not involve any specification of the property in virtue of which boys do not cry. Even if the speaker has in mind some such "in virtue of" property, it is not an integral part of his assertion, and the listeners are not committed to accommodated it. All he claims is that "this is not accidental", i.e. not limited to actual boys and actual situations only.

As said above, this intuitive difference between the "in virtue of" and descriptive generalizations expressed by IS and BP sentence can be fruitfully and formally captured by positing two different accessibility relations which can restrict the modalized Gen operators. The next two sections illustrate how this can be done.

\subsection{The "in virtue of" accessibility relation of IS sentences}

Following Kratzer 1981 ideas about circumstantial modality, and Brennan's 1993 work on modal verbs with root interpretation, I will take the basic accessibility relation of IS sentences to be systematically restricted by the "in virtue of" property. Specifically, the worlds we quantify over are those where every individual with the subject property has the "in virtue of" property.

Suppose you hear for example (34a), and the "in virtue of" property you accommodate is "being tough". In this case the accessible worlds are those where every boy is tough, so the sentence has the truth conditions in (34b):

a. A boy does not cry (in virtue of $\wedge^{\wedge}$ be tough)

b. $\forall \mathrm{w}^{\prime}\left[\forall \mathrm{x}\left[\right.\right.$ boy $\left.\left.\left(\mathrm{x}, \mathrm{w}^{\prime}\right)\right] \rightarrow\left[\operatorname{tough}\left(\mathrm{x}, \mathrm{w}^{\prime}\right)\right]\right] \rightarrow$

$$
\left[\forall \mathrm{x}, \mathrm{s}\left[\mathrm{boy}\left(\mathrm{x}, \mathrm{w}^{\prime}\right) \wedge \mathrm{C}\left(\mathrm{s}, \mathrm{x}, \mathrm{w}^{\prime}\right)\right] \rightarrow\left[\neg \mathrm{cry}\left(\mathrm{s}, \mathrm{x}, \mathrm{w}^{\prime}\right)\right]\right]
$$

Paraphrase: In all worlds where every boy is tough, every boy (in all relevant situations) doesn't cry.

Similarly, if you hear (35a), and accommodate "in virtue of having a four legged genetic makeup", the sentence will have the truth conditions in (35b):

a. A dog has four legs (in virtue of ${ }^{\wedge}$ has a 4 legged genetic makeup)

b. $\forall \mathrm{w}^{\prime}\left[\forall \mathrm{x}\left[\operatorname{dog}\left(\mathrm{x}, \mathrm{w}^{\prime}\right)\right] \rightarrow\left[\right.\right.$ has a 4 legged genetic makeup $\left.\left.\left(\mathrm{x}, \mathrm{w}^{\prime}\right)\right]\right] \rightarrow$ $\left[\forall \mathrm{x}, \mathrm{s}\left[\operatorname{dog}\left(\mathrm{x}, \mathrm{w}^{\prime}\right) \wedge \mathrm{C}\left(\mathrm{s}, \mathrm{x}, \mathrm{w}^{\prime}\right)\right] \rightarrow\left[\right.\right.$ has 4 legs $\left.\left.\left(\mathrm{s}, \mathrm{x}, \mathrm{w}^{\prime}\right)\right]\right]$

In general, then, if we take the subject property to be ${ }^{\wedge} \mathrm{P}$, the VP property to be ${ }^{\wedge} \mathrm{Q}$ and the chosen "in virtue of" property to be ${ }^{\wedge} \mathrm{S}$, the truth conditions of an IS sentences seem to be the ones in (36) 


$$
\begin{aligned}
& \text { An IS sentence is true in w iff: } \\
& \forall \mathrm{w}^{\prime}\left[\forall \mathrm{x}\left[\mathrm{P}\left(\mathrm{x}, \mathrm{w}^{\prime}\right)\right] \rightarrow\left[\mathrm{S}\left(\mathrm{x} . \mathrm{w}^{\prime}\right)\right]\right] \rightarrow \\
& \quad\left[\forall \mathrm{x}, \mathrm{s}\left[\mathrm{P}\left(\mathrm{x}, \mathrm{w}^{\prime}\right) \wedge \mathrm{C}\left(\mathrm{s}, \mathrm{x}, \mathrm{w}^{\prime}\right)\right] \rightarrow\left[\mathrm{Q}\left(\mathrm{s}, \mathrm{x}, \mathrm{w}^{\prime}\right)\right]\right]
\end{aligned}
$$

This, however, is clearly not enough, because of two related problems. Firstly, the accessibility relation in (36) does not specify at all how the worlds quantified over ( $w^{\prime}$ in (36)) are accessible from the world of evaluation $w$, and after all, this is what we want to show in an accessibility relation. In addition, there is no limitation, or restriction in (36) on the choice of the "in virtue of", "S property, so in principle any arbitrary or even far fetched property can be chosen. This, however, may wrongly lead all IS sentences to be true. A clearly false sentence like (37a), for example, may be interpreted as in (37b), and thus wrongly predicted to be true:

a. A dog has three legs

b. "In all worlds where every dog has a three legged genetic makeup, every dog has three legs"

The solution to both these problems is the same. In accommodating an "in virtue of" property, we cannot really choose any arbitrary, far-fetched property we want. The property we choose has to meet some criteria imposed by our real world knowledge. For a start I assume that we can only choose those properties which, given our knowledge of the actual world, are "associated" with the subject property.

We should now define precisely the nature of the "association" relation between two properties, relative to a world $\mathrm{w}$. Take for example the property of being a boy. In our world we associate with it a number of properties, e.g. being male, loving sports, being tough, etc. Clearly not all such properties are true of all boys in the actual world. In actuality, for example, only some (maybe half) of the boys love sports, and only a minority of the boys are really tough. On the other hand, we will not associate with the property of being a judge something like "hating tomatoes", even if it happens to be the case that all actual judges hate tomatoes. What determines, then, whether a certain property is associated with another property in our world is not the actual state of affairs, in the actual world, but rather our beliefs, norms, stereotypes, etc. about the actual world. We associate being tough with being a boy, for example, since it follows from the norms in our actual world that every boy is tough. I.e. the universal statement "every boy is tough" holds, not in the actual worlds itself, but in all worlds which are normatively accessible from it. In still other words, this universal statement is a (normative) necessity in $\mathrm{w}$. These considerations lead to the definition in (38):

(38) Formal definition of "associated properties":

${ }^{\wedge} \mathrm{S}$ is associated with ${ }^{\wedge} \mathrm{P}$ in $\mathrm{w}$ iff there is a "Kratzerian", accessibility function $f$ from worlds to sets of propositions (e.g. epistemic, deontic, stereotypical, legal, etc.) s.t. $\forall \mathrm{w}^{\prime \prime}\left[\mathrm{w}^{\prime \prime} \mathrm{R}_{f} \mathrm{w}\right] \rightarrow\left[\forall \mathrm{x}\left[\mathrm{P}\left(\mathrm{x}, \mathrm{w}^{\prime \prime}\right)\right] \rightarrow\left[\mathrm{S}\left(\mathrm{x}, \mathrm{w}^{\prime \prime}\right)\right]\right]$

Paraphrase: ${ }^{\wedge} S$ is associated with ${ }^{\wedge} P$ in $w$ iff $\forall x P(x) \rightarrow S(x)$ holds in all worlds epistemically accessible from $w$ (where the facts known in $w$ hold), OR deontically accessible from $w$ (where what is commanded in w holds), OR stereotypically accessible from $\mathrm{w}$ - (where the stereotypes in w hold), etc. 
Integrating this definition of associated properties into the truth conditions of IS sentences, what we get is something like (39):

An IS sentence is true in $w$ iff:

$\exists^{\wedge} \mathrm{S} \forall \mathrm{w}^{\prime}\left[\forall \mathrm{x}\left[\mathrm{P}\left(\mathrm{x}, \mathrm{w}^{\prime}\right)\right] \rightarrow\left[\mathrm{S}\left(\mathrm{x} . \mathrm{w}^{\prime}\right) \wedge \wedge\right.\right.$ S is associated in $\mathrm{w}$ with $\left.\left.{ }^{\wedge} \mathrm{P}\right]\right] \rightarrow$ $\left[\forall \mathrm{x}, \mathrm{s}\left[\mathrm{P}\left(\mathrm{x}, \mathrm{w}^{\prime}\right) \wedge \mathrm{C}\left(\mathrm{s}, \mathrm{x}, \mathrm{w}^{\prime}\right)\right] \rightarrow\left[\mathrm{Q}\left(\mathrm{s}, \mathrm{x}, \mathrm{w}^{\prime}\right)\right]\right]$

Paraphrase: An IS sentence is true in a world $w$ iff there is a property ${ }^{\wedge} S$, s.t. in all worlds $\mathrm{W}^{\prime}$, where every member of the subject set $(\mathrm{P})$ has $\mathrm{S}$, and, furthermore, $\wedge \mathrm{S}$ is associated in $\mathrm{W}$ with the ${ }^{\wedge} \mathrm{P}$ property (given (38)), then every member of the subject set, in all relevant situations, is a member of the VP set (Q) as well.

The IS sentence in (37a), for example, will have now the truth conditions in (40), instead of (37b):

(40) There is a property ${ }^{\wedge} \mathrm{S}$, s.t. in all worlds where every $\operatorname{dog}$ has $\mathrm{S}$, and this property ${ }^{\wedge} \mathrm{S}$ is associated in $\mathrm{w}$ with being a dog, every dog in any situation has three legs

Now there are many properties we associate in our world with being a dog: having a tail, having a 4 legged genetic makeup, being loyal, barking, etc. Crucially, however, neither of them leads all dogs to have 3 legs. Thus, we correctly predict that this sentence is false.

Notice also that adding the "association" requirements into the truth conditions means that the semantics of IS sentences involves, in fact, two levels of modality: The basic set of accessible worlds (w' in (39)) is defined as those in which "every $P$ has $S$ " holds. But the choice of the "in virtue of" $\wedge \mathrm{S}$, property depends, in turn, on a more traditional, "Kratzerian" accessibility relation, which gives a set of worlds where what is known, commanded, etc. in w holds ( $w^{\prime \prime}$ in the (38)). Put in other words, in order to guarantee that the "in virtue of" $\wedge \mathrm{S}$ property is not an arbitrary, far fetched property from the point of view of our real world knowledge, we require that the basic set of accessible worlds w' we quantify over (where "every P is S" holds) is a superset of the set of worlds which are epistemically, deontically, stereotypically etc. accessible from the world of evaluation $w$.

This "double modality" captures the intuition that a speaker of an IS sentence not only asserts that a certain generalization is nonaccidental (i.e. say that "every $P$ is Q" holds in a set of accessible worlds w'), but also that it is true in virtue of another generalization - "every $\mathrm{P}$ is $\mathrm{S}$ " - which is itself nonaccidental (i.e. a necessity) w.r.t. our world.

\subsection{The accessibility relation of "descriptive" generalizations}

As claimed above, BP sentences can express "in virtue of" generalizations, just like their IS counterparts, thus having truth conditions as in (39) above. Unlike IS sentences, however, they can also express "descriptive" generalizations, where no "in virtue of" property is involved. The descriptive reading of (41a), for example, can be paraphrased as in (41b):

a. Librarians whose names end with ' $t$ ' speak very quickly 
b. The generalization "every librarian whose name ends with ' $t$ ' speaks very quickly" is nonaccidental - not limited to the actual world - but is expected to hold in other, nonactual worlds as well.

The question we need to answer now is what are these accessible worlds ? Specifically, in which aspects are they like our actual worlds? Which elements of the actual worlds are "copied" into them ?

The answer I suggest is that since, unlike what happens with "in virtue of" generalizations, with descriptive ones do not specify (or even know) in virtue of what the generalization is true, we don't specify (or even know) what actual-world elements are "copied" into the accessible worlds. Put in other words, the accessibility relation remains vague.

I suggest to formally capture this vagueness using Lewis's 1986 overall, maximal similarity, which is inherently vague. The truth conditions of a descriptive generic like (41a), for example, will be those in (42):

$$
\begin{aligned}
& \forall \mathrm{w}^{\prime}\left[\mathrm{Max}\left(\mathrm{w}^{\prime}, \mathrm{w}\right)\right] \rightarrow \\
& \quad\left[\forall \mathrm{x}, \mathrm{s}\left[\text { librarians whose names end with ' } \mathrm{t} \text { ' }\left(\mathrm{x}, \mathrm{w}^{\prime}\right) \wedge \mathrm{C}\left(\mathrm{s}, \mathrm{x}, \mathrm{w}^{\prime}\right)\right] \rightarrow\right. \text { [speak } \\
& \left.\left.\quad \text { quickly }\left(\mathrm{s}, \mathrm{x}, \mathrm{w}^{\prime}\right)\right]\right] \\
& \text { where } \operatorname{Max}\left(\mathrm{w}^{\prime}, \mathrm{w}\right) \text { holds iff } \mathrm{w}^{\prime} \text { ' is maximally similar to } \mathrm{w} \text { except from what } \\
& \text { is needed to possibly allow for the existence of a different population of (e.g. } \\
& \text { more, less, or totally different) librarians whose names end with ' } \mathrm{t} \text { '. }
\end{aligned}
$$

\section{Accounting for differences between minimal pairs of IS and BP sentences.}

We can now see how the two distinct accessibility relations help accounting for the differences between minimally contrasting IS and BP sentences we looked at above.

Firstly, the stronger vs. weaker "law-likeness" expressed by IS and BP sentences, respectively, reported by Burton Roberts 1977, result from the different degree of similarity between the accessible worlds and the actual one, in the two types of accessibility relations. IS sentences are felt to express strong rules since the universal generalization over individuals and situations is required to hold in worlds which preserve a very specific and narrow aspect of the actual world: those where a certain epistemic, deontic stereotypical, etc. necessity about the actual world (of the form "every P member has S) holds. On the other hand, although in descriptive BP sentences the universal generalization has to hold, not merely in the actual world, but in a set of possible worlds, these possible worlds preserve a much wider range of characteristics of the actual world. These are those worlds which are maximally similar to $\mathrm{w}$ (except from possibly having other $\mathrm{P}$ members) This "wide perspective" similarity to the actual world is what leads to the double nature of descriptive BP sentences: expressing nonaccidental generalizations, on the one hand, which on the other hand are also "descriptive" and more about actuality than the generalizations expressed by corresponding IS sentences.

We can now also show why IS sentences like (43a), with subject expressing "extremely unnatural classes", have a salient existential reading, and are odd as generic. Given our claims in section (4.2), (43a) will have truth conditions as in (43b):
a. \# A Norwegian student whose name ends with ' $s$ ' or " wears thick green socks


b. $\exists^{\wedge} \mathrm{S} \forall \mathrm{w}^{\prime}[\forall \mathrm{x}$ [Norwegian student whose name ends with 's' or ' $\mathrm{g}$ '( $\left.\mathrm{x})\right]$

$\rightarrow\left[\mathrm{S}\left(\mathrm{x}, \mathrm{w}^{\prime}\right) \wedge{ }^{\wedge} \mathrm{S}\right.$ is associated in $\mathrm{w}$ with ${ }^{\wedge}$ Norwegian student whose name ends with 's' or ' $\mathrm{g}$ ']] $\rightarrow$

$[\forall \mathrm{x}, \mathrm{s}$ [Norwegian student whose name ends with 's' or ' $\mathrm{g}$ ' $(\mathrm{x}, \mathrm{w}$ ') $\wedge$

$\left.\mathrm{C}\left(\mathrm{s}, \mathrm{x}, \mathrm{w}^{\prime}\right)\right] \rightarrow\left[\right.$ wear thick green socks $\left.\left.\left(\mathrm{s}, \mathrm{X}, \mathrm{w}^{\prime}\right)\right]\right]$

paraphrase: $(43 \mathrm{a})$ is true in $\mathrm{w}$ iff there is an ${ }^{\wedge} \mathrm{S}$ property s.t. in all worlds where every Norwegian Student whose name ends with ' $s$ ' or ' $g$ ' has ${ }^{\wedge} S$, and ${ }^{\wedge} \mathrm{S}$ is associated in $\mathrm{w}$ with being a Norwegian Student whose name ends with ' $s$ ' or ' $g$ ', every such Norwegian student, in all relevant situations, wears thick green sock

Unlike what happens with e.g. A dog has three legs, where there were many properties we could associate with the subject property ${ }^{\wedge} \mathrm{P}$, but none lead to having the VP property $(\wedge \mathrm{Q})$, here the problem is much more serious. Given our real world knowledge there is, in fact, no (nontrivial) property which we associate with being a Norwegian student whose name ends with ' $s$ ' or ' $g$ '. This is because the universal statement $\forall x$ [Norwegian student whose name ends with ' $s$ ' or ' $\mathrm{g}$ ' $(\mathrm{x})][\mathrm{S}(\mathrm{x})]$ does not follow from any set of shared known facts, norms, stereotypes etc., since in our world we have no shared knowledge, norms, stereotypes etc. on being a Norwegian student whose name ends with ' $s$ ' or ' $g$ '. In fact, this is exactly what makes such properties to feel so "unnatural".

But if no property is associated with being a Norwegian student whose name ends with ' $s$ ' or ' $g$ ', then no accessible worlds can be found in which to evaluate the generalization over individuals and situations. In such a case, however, a presupposition that accessible worlds w' exist, which is (conventionally) triggered by the universal quantification over worlds, is not met, and this leads to the infelicity of (43a), as generic, and to the salient existential reading of the subject ${ }^{7}$.

Notice, however, that the "association" requirement on the choice of the "in virtue of", $\wedge \mathrm{S}$ property is still not enough to explain why IS sentences as in (44) mentioned above, are infelicitous, and not merely false like those in (45):
a. \# A man is blond
b. \# An uncle is garnulous
c. \# An Italian restaurant is closed tonight.
a. A dog has three legs
b. A Pentium VI computer works very slowly
c. A pine tree has wide leaves

The problem is that the association requirement is equally met in both (44) and (45). This because (unlike being, e.g. a Norwegian student whose name ends with ' $\mathrm{s}$ ' or ' $g$ ') being an uncle, a man, or an Italian restaurant, just like being a dog, a Pentium computer or a pine tree - are very natural properties. They are natural in the sense that we associate many properties with them.

I would like to suggest that what leads to the infelicity of the IS sentences in (44) is the failure of an additional presuppositional requirement on the choice and accommodation of the "in virtue of", $\wedge \mathrm{S}$ property. Intuitively, the idea is that besides being not too far-fetched w.r.t. our real world knowledge about the subject property, the "in virtue of", $\wedge \mathrm{S}$ property has to be not too far-fetched w.r.t. our real world knowledge about the VP property $\left({ }^{\wedge} \mathrm{Q}\right)$ as well. Specifically, besides being associated 
with ${ }^{\wedge} \mathrm{P}$ in $\mathrm{w}$, the chosen ${ }^{\wedge} \mathrm{S}$ property should be perceived as a reasonable causer for properties of the sort of ${ }^{\wedge} Q^{8}$. This can be phrased more precisely as in (46):

The additional presuppositional requirement on the choice of ${ }^{\wedge} \mathrm{S}$

${ }^{\wedge} \mathrm{S}$ is s.t. there is a good possibility (relative to $\mathrm{w}$ ) that

$[\forall \mathrm{x}[\mathrm{P}(\mathrm{x}) \wedge \mathrm{S}(\mathrm{x})] \rightarrow \mathrm{Q}(\mathrm{x})] \vee[\forall \mathrm{x}[\mathrm{P}(\mathrm{x}) \wedge \mathrm{S}(\mathrm{x})] \rightarrow \neg \mathrm{Q}(\mathrm{x})]$

Paraphrase: ${ }^{\wedge} \mathrm{S}$ is s.t. there is a good possibility, from the point of view of our world, that either every $\mathrm{P}$ individual with $\mathrm{S}$ has $\mathrm{Q}$, or every $\mathrm{P}$ individual with $\mathrm{S}$ does not have $\mathrm{Q}$.

Compare, for example (45a) (A dog has three legs) and (44a) (A man is

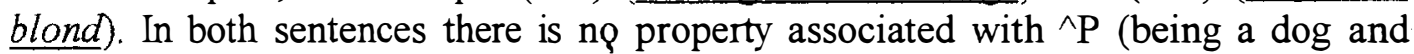
being a man) which is taken to be a "reasonable causer" of the ${ }^{\wedge} \mathrm{Q}$ property itself (i.e. of having three legs, and being blond, respectively). Nonetheless, when we think about properties of the sort of ${ }^{\wedge} \mathrm{Q}$, we can see that there is a difference between the two sentences: We can easily find a property associated with being a dog which is a reasonable causer for having a certain number of legs (i.e. a property of the sort of "having three legs"), namely having the regular genetic makeup for a certain, specific, and fixed number of legs. In contrast, among all the properties we associate with being a man there is even no property which we take to reasonably lead all men to be blond, or all men not to be blond, i.e. to reasonably lead to having a certain specific and fixed hair color, or any other property of the sort of "being blond". Similarly, among all properties we associate with being an Italian restaurant, none reasonably leads to properties of the sort of "being closed on the night when the sentence is uttered". This is why (44c) is also infelicitous as generic.

We can now show that, unlike Lawler's 1973 essential / nonessential distinction, the combination of the two pragmatic-based presuppositional requirements on the choice of the "in virtue of", $\wedge \mathrm{S}$ property indeed "slices the cake" correctly: All infelicitous IS sentences are those where (at least) one of the requirements on the "in virtue of", ^S property isn't met: being associated, from the point of view of $\mathrm{w}$, with the subject property $(\wedge \mathrm{P})$, or being a reasonable causer, from the point of view of $w$, of properties of the sort of the VP property $(\wedge \mathrm{Q})^{9}$.

Unlike IS sentences like (43a) or (44a-c), minimally contrasting BP ones (as in (12), (13) and (15) above) are perfectly felicitous as generic. This is because they can express "descriptive" generalizations, where the characterization of accessible worlds does involve any "in virtue of" property, and thus crucially, does not depend at all on satisfying real world knowledge requirements and assumptions concerning the subject and VP property. For example, even if the BP subject expresses an extremely unnatural property (as in (13a) and (13b) above), and no property can be associated with it, we can still easily find accessible worlds (those maximally similar to w except from possible having other $\mathrm{P}$ members) in which to evaluate the generalization, and thus such sentences are perfectly felicitous. Similarly, BP sentences like Men are blond I Uncles are garrulous etc. are perfectly fine as generic, since on the descriptive reading we don't care that there is no property, associated with the subject property, which also reasonably leads to properties of the sort of the VP property. This pragmatic based requirement is irrelevant to the definition of the accessibility relation. Thus, unlike IS sentences, BP ones can express the most bizarre generalizations, and still be felicitous. 


\section{The big "why" question: How to explain the difference in availability of accessibility relations?}

The main claim made until now is that the variety of semantic, pragmatic and distributional differences between minimally contrasting IS and BP sentences result from the difference in availability of accessibility relations, which can be summarized in (47):

a. The generic quantifier of IS sentences is only compatible with an "in virtue of" accessibility relation.

b. The generic quantifier of BP sentences is compatible with both an "in virtue of" and a "descriptive" accessibility relation.

The obvious question to ask now, is how to explain this difference in availability of accessibility relations itself. In particular, how is this difference in the semantics of the two types of sentences (in terms of the different accessibility relations available) can be related to, and ideally derived from, the only surface difference between the two types of sentences, namely the distinction between the indefinite singular and bare plural NPs in these sentences?

A preliminary, but nonetheless promising direction to take in answering this question is the following: Until now we followed the standard approach (e.g. Chierchia 1995, ter-Meulen 1995, Wilkinson 1991, Link 1995) according to which IS NPs denote property expressions, whereas BP NPs are ambiguous: they denote property expressions in quantificational - modal (I-) generic sentences (like Dogs bark), and kind referring terms in Direct Kind Predication generics (e.g. Dodos are extinct / Rats reached Australia in 1770). Consequently, aside from the accessibility relation, minimally contrasting IS and BP sentences (like $A$ dog barks and Dogs bark) were represented equally, as in (48):

$$
\forall \mathrm{w}^{\prime} \underbrace{\left[\forall \mathrm{x}, \mathrm{s}\left[\operatorname{dog}\left(\mathrm{x}, \mathrm{w}^{\prime}\right) \wedge \mathrm{C}\left(\mathrm{s}, \mathrm{x}, \mathrm{w}^{\prime}\right)\right] \rightarrow\left[\operatorname{bark}\left(\mathrm{s}, \mathrm{x}, \mathrm{w}^{\prime}\right)\right]\right]}_{\left[\mathrm{w}^{\prime} \text { is appropriately accessible from } \mathrm{w}\right]}
$$

To start explaining the difference in availability of accessibility relations, however, we can now make use of an alternative view (e.g. Krifka et al 1995, Chierhica 1998, Delfitto 1997, Cohen 1999)), according to which BP NPs can (or even must) denote kinds also in quantificational/modal generics like Dogs have four legs / Men are blond / Boys don't cry etc. Adopting such a view we will continue to represent both such IS and BP sentences as quantificational, modalized tripartite structure. However, whereas with IS sentences the restriction of Gen continues to contain a property expression, with BP ones the restriction of Gen will contain a kind expression.

There are several possible ways as to how to integrate a kind referring term in to the quantificational-modal structure of BP sentences. One possibility, in the spirit of von Fintel 1994, is to take BP sentences to express quantification over minimal situations which involve existentially quantified individuals realizing the kind, as in (49):

$$
\text { Gen s }\left[\exists \mathrm{x} R(\mathrm{x}, \mathrm{DOGS}) \wedge \text { Involve }_{\min }(\mathrm{s}, \mathrm{x})\right] \leq\left[\exists \mathrm{xR}(\mathrm{x}, \mathrm{DOGS}) \wedge \operatorname{bark}_{\min }(\mathrm{s}, \mathrm{x})\right]
$$
Paraphrase: In all accessible worlds every minimal situation, involving 
some individual realizing the kind "dogs", is part of (or - can be extended to) a minimal barking situation involving some realization of the kind "dogs"

Another possibility is to assume that BP sentences involve "Modalized Kind Predication" (in Delfitto's 1997 terminology), as in (50):

$\lambda x$. Gen s [Involve $(\mathrm{s}, \mathrm{x})] \rightarrow[$ bark $(\mathrm{s}, \mathrm{x})](\mathrm{DOGS})]$

Paraphrase The set of individuals for which, every relevant situation in all accessible worlds is a barking situation, has the kind "dogs" as a member.

A third, similar, possibility is to take BP sentences to involve quantification over all situations involving the kind (as in (51a)), and assume that at this stage the accessibility relation is determined. Only after the accessibility relation is fixed the kind term is typed shifted into the set of realizations of the kind (as in Chierchia's 1998, Krifka et al's 1995 and Cohen's 1999 suggestions), so we end up quantifying over all situations involving a realization of the kind dog (as in (51b)):
a. Gen s $[\mathrm{C}(\mathrm{s}, \mathrm{DOGS})] \rightarrow[\operatorname{bark}(\mathrm{s}, \mathrm{DOGS})]]$ Paraphrase: Any contextually relevant situation involving the kind "dogs" is a barking situation (in all accessible worlds).
b. $\forall \mathrm{x}, \mathrm{s}[\mathrm{R}(\mathrm{x}, \mathrm{DOGS}) \wedge \mathrm{C}(\mathrm{s}, \mathrm{x})] \rightarrow[$ bark $(\mathrm{s}, \mathrm{x})]$ Paraphrase: Any contextually relevant situation involving any instance of the dog kind is a barking situation (in all accessible worlds).

I will not now attempt to decide which of these possibilities is the best. The assumption which stands behind all of them is that it is the presence of the kind term in the restriction of Gen, rather than a property expression, which somehow leads to the difference in availability of accessibility relations. But how exactly?

The answer to this question lies in a closer examination of the linguistic behavior of kind terms. No matter whether one takes kinds to be basic entities in the model (like Carlson 1977, and Cohen 1999), or some type of intensional group entities (as Chierchia 1998 and Dobrovie Sorin \& Laca 1996), the relevant point for the present discussion is that, as convincingly shown already by Carlson 1977 (and more recently by Chierchia 1998), kind denoting NPs pattern like individual denoting NPs, i.e. proper names and definite descriptions, in a variety of diagnostical constructions (w.r.t. to scope ambiguities, in the so called "so called" construction, etc.). The crucial point for us is that this similarity seems to hold also with respect to the type of modality or "nonaccidentalness" involved.

For example, Carlson 1995 claims that the salient reading of classic habituals with proper name subjects, as in (52a), usually represented as in (52b), is that of a "weak descriptive generalization". In our terms, sentences like (52a) have a "descriptive" accessibility relation:
a. John walks to school
b. Gen s $[\mathrm{C}(\mathrm{s}, \mathrm{j})] \rightarrow[$ walk to school $(\mathrm{s}, \mathrm{j})]$

There are also habituals with proper name subjects which are ambiguous between the "in virtue of" and the "descriptive" accessibility relations, just like many 
BP sentences. For example the classic (53), can either have the "in virtue of" reading paraphrased in (54a), or the descriptive one, paraphrased in (54b):

(53) Mary handles the mail form Antarctica

(54) a. "In virtue of her job contract Mary is handling mail from Antarctica in all relevant situations"

b. "That Mary is handling mail from Antarctica in all relevant situations is not accidental (with no specification of the "in virtue of" factor)"

What seems to emerge from this comparison between BP sentences and habituals with proper name subjects is the generalization in (55):

a. When Gen is restricted by a property expression (as in $\underline{A d o g}$ barks) only the "in virtue of" accessibility relation is available

b. When Gen is restricted by an individual expression (as in Dogs bark / Fido barks) both the "in virtue of" and "descriptive" accessibility relations are available.

But what is the reason for this correlation between the property / individual nature of the element restricting Gen, and the "in virtue of" / "descriptive" accessibility relations (which restrict the set of worlds we quantify over) ?

A possible preliminary answer to this question is based on two further assumptions. The first is that with natural language generics the characterization of the accessibility relation is made under the assumption that the entity restricting Gen is a natural entity. The second is that there is a significant difference between what we perceive to be natural properties, and natural individuals.

Intuitively, properties are considered natural only to the extent we have some pre-knowledge about them, i.e. if we associate other properties with them. Put in other words, natural properties systematically "bring with them" associated properties. In contrast, individuals can be considered perfectly natural even if we have no preknowledge, stereotypes, norms etc. associated with them. This idea seems rather reasonable when we think about individuals referred to by proper name (like "Terner" - even if one associates no property with the individual denoted by this name, it will not be perceived as an unnatural individual). Crucially, however, I believe it holds even when the individual is referred to by definite descriptions (like The tall man with the blue hat the spy) or by kind referring bare plurals (dogs, blond uncles with six names). Although these latter descriptions contain predicate expressions, of which we naturally have real world knowledge, their use is only to help pick out an individual (object or kind level). In Donnelan's 1966 terms, these referring expressions are used, or at least can be used, referentially and not attributively.

Notice that I am not claiming that natural individuals cannot be associated with properties, only that, unlike what happens with properties, such an association is not necessarily needed for considering them natural. Thus, many people may associate various properties with the individuals denoted by the proper name and definite descriptive "Shakespeare", and "The man who works in this office", respectively. Crucially, however, even a person who has no idea who Shakespeare was, or a person who knows nothing about this office, will have no problem considering the individuals denoted by these NPs "natural individuals". In the case of properties, on the other hand, the association of properties is necessary, and systematically required, in order to consider them natural. E.g. the property "being a brown haired person born 
at the beginning of July" is considered so unnatural exactly because we associated with it no (nontrivial) other properties.

Now, when uttering a characterizing generic, we are making a nonaccidental generalization. I.e. we are claiming that a certain generalization holds in other possible worlds, accessible from the actual one. From the point of view of generics, the decision on which worlds will be considered accessible (i.e. in which worlds we will evaluate the generalization) is sensitive to the kind of natural entity - individual or property - that the generalization is about.

Since natural properties (denoted by IS subjects) systematically come with their associated properties, i.e. some real world knowledge about them is systematically and prominently present, the similarity of the accessible worlds to ours systematically makes use of this assumed real world knowledge, and defines the worlds similar to ours in terms of some shared real world knowledge associated with the property restricting the generic operator (as in the "in virtue of" accessibility relation). In contrast, since natural individuals (denoted by proper names, definite descriptions and BP NPs) are not systematically associated with properties, the decision on the type of similarity of accessible worlds to our world cannot rely on such associated properties, and hence can always be defined in a default and vague way, as with the "descriptive" accessibility relation. In addition, in many cases, where it is reasonable to associate "in virtue of" properties with the kind denoted by the BP subject, the "in virtue of" accessibility relation is also available.

These suggestions are, of course, very intuitive and preliminary. Further research should now continue to check whether and how they can be independently motivated and more precisely formulated.

\section{Summary}

The main claims made in this paper can be summarized as follows:

(*) In addition to the classic kind / characteristics (D/I) genericity distinction there is another distinction in the generic domain between two types of characterizing, I- generics, expressing "In virtue of" and "descriptive" generalizations

(*) This distinction is syntactically encoded in English: BP sentences can express both types of generalizations, whereas IS sentences can only express the former ones.

(*) Both types of sentences express nonaccidental generalizations over individuals and situations, and have a basically equivalent tripartite quantificational modalized structure : this accounts for the strong similarities between the two types of sentences.

$\left(^{*}\right)$ The two types of generalizations differ in the type of accessibility relation restricting the modalized Gen operator, i.e. in the choice of accessible worlds where the generalizations are evaluated. These differences between the accessibility relations - in terms of the degree of similarity of accessible worlds to the actual one, and the presence vs. absence of real world knowledge requirements in the characterization of accessible worlds - account for the semantic, pragmatic and distributional differences between minimally contrasting IS and BP sentences, which until now did not get a satisfactory characterization / account. 
(*) A promising direction to explain the difference in availability of accessibility relations between IS and BP sentences, is to derive it from the interpretation of the entity restricting Gen: a property expression (in the case of IS sentences), or a kind referring expression (in the case of BP sentences), while assuming a systematic difference between natural properties and natural individuals. This direction is supported by the similar type of modality expressed by both BP sentences and simple habitual sentences with proper name subjects.

\section{Endnotes}

* I want to deeply thank Susan Rothstein for her invaluable help and support, professional and other, in the various stages of working on this material, and. Gennaro Chierchia, Greg Carlson, Angelika Kratzer and Fred Landman for their time, patience and the numerous helpful comments and suggestions I got from them. Many thanks also to Maria Bittner, Veneeta Dayal, Edit Doron, Manfred Krifka, Anita Mittwoch, and especially Ariel Cohen for their help and suggestions, and to the audiences of the IATL 1997, SALT 2002, and Chorons 2002 conferences, as well as the Hebrew university research group for logic and language, for their helpful comments.

${ }^{1}$ Following Chierhchia 1995 I assume here that we get exactly the same structure, with quantification over situations, also when the VP expresses an individual-level property, e.g. with $A$ dog has four legs and Dogs have four legs.

${ }^{2}$ The tolerance of exceptions of both IS and BP sentences can be captured by further restricting the set of worlds by some "normal" /"stereotypical" ordering source (as suggested by Krifka et al 1995), or by further restricting the set of individuals (and situations) by some "domain vague" restriction, (e.g. as in Kadmon \& Landman's 1993 suggestion, developed in Greenberg 2002). The exact choice of the mechanism for tolerating exceptions is not crucial for the claims I make below.

${ }^{3}$ In Greenberg 1998 and 2002 I showed that temporally restricted BP sentences as in (16) are indeed (I-) generic, and not e.g. "quasi-universal" (in Condoravdi's 1996 terms). E.g. they support counterfactuals, tolerate legitimate exceptions and obey Cohen's 1999 "homogeneity constraint", just like standard (I-) generics like Dogs have four legs.

${ }^{4}$ Similar suggestions are found in Dobrovie-Sorin \& laca 1996 and Cohen 2001.

${ }^{5}$ But not only in English. A similar semantic distinction seems to be manifested in the syntax of other languages, e.g. in French - through the distinction between sentences with the determiner les and those with $\underline{\text { des }}$ (see de Swart 1994 who quotes in this respect Calier 1989), and, as pointed out to me by Greg Carlson (p.c.), also in some African languages, e.g. Swahili, through the distinction between generic sentences with and without an overt marker $h u$.

${ }^{6}$ The distinction between these two types of generalizations is similar to, and to a large extent inspired by Carlson's 1995 intuitive distinction between the "rules and regulations" and the "inductive" approaches to genericity. Carlson, however, suggested we should choose which of these approaches is better in analyzing generic sentences. In contrast, my claim is that there is no need to choose, since both types of generalizations are expressed in natural language. (See also Cohen 2001 for a similar claim, and Greenberg 2002 for a discussion of the differences between Cohen's theory and the present one). 
${ }^{7}$ Another possible way to phrase the problem is to say that if the requirement that

" $\mathrm{S}$ is associated with $\wedge \mathrm{P}$ in $\mathrm{w}$ " isn't met, the whole antecedent of the universal quantification over worlds is false, and thus the domain of accessible worlds we universally quantify over is empty. This leads to the trivial truth of the whole universal quantification, which in turn leads to infelicity. Notice that a similar thing happens not only with universal quantification over worlds, but also with universal quantification over individuals, as shown, e.g. in de Jong and Verkuyl 1984. E.g. the sentence Every male student in my class got above 80 is infelicitous if the domain of male students in my class is empty.

${ }^{8}$ In Greenberg 2002 I show that this additional presupposition need not be stipulated, but is conversationally triggered by a combination of the existing truth conditions of IS sentences and the Grician maxim of quality.

${ }^{9}$ In this sense, the two restrictions on ${ }^{\wedge} \mathrm{S}$ formally capture Chierchia's 1995 intuition concerning the double restriction on the set of conditions ("felicity conditions") which define the accessible worlds of generics:

"Actually, it seems intuitively that there are two kinds of contextual restrictions [on the set of felicity conditions]. One comes, as it were, from the verb; it determines what conditions have to be met for an individual to undertake an action. The other comes from the noun; it determines what features of the species are relevant for the action." (p.196)

\section{References.}

Brennan, V. (1993), "Root and Epistemic Modal Auxiliary Verbs", Ph.D. dissertation, Umass., Amherst.

Burton-Roberts, N. (1977), "Generic Sentences and Analyticity”, Studies in

Language 1:155-196.

Carlier, A. (1989), "Genericite du syntagme nominal sujet et modalites" Travaux de Linguistique 19: 33-56.

Carlson, G. (1977), "Reference to Kinds in English", Ph.D. dissertation, Umass., Amherst.

Carlson, G. (1995), "Truth Conditions of Generic Sentences: Two Contrasting Views" in G. Carlson and F.J. Pelletier (eds.) The Generic Book, The University of Chicago Press, Chicago.

Chierchia, G. (1995) "Individual Level Predicates as Inherent Generics" in G. Carlson and F.J. Pelletier (eds.) The Generic

Book, The University of Chicago Press, Chicago.

Chierchia, G. (1998), "Reference to Kinds Across Languages", Natural Language and Linguistic Theory 6:339-405.

Cohen, A. (1999) “Generics, Frequency Adverbs and Probability". Linguistics and Philosophy 22:221-253.

Cohen, A. (2001) "On the Generic Use of Indefinite Singulars". Journal of Semantics 18:183-209.

Condoravdi, C. (1993), "Weekly and Strongly Novel Noun Phrases", in Proceedings of the 11th. WCCFL.

Dahl, O, (1975), "On Generics”, in E. Keenan, ed., Formal Semantics of Natural

Language, 99-111. Cambridge University Press, Cambridge.

Delfitto, D. (1997) "Aspect, Genericity and Bare Plurals" ms. Utrecht University. 
Dobrovie Sorin, C. and B. Laca (1996) "Generic Bare NPs", ms. Universite Paris 7 and Universite de Strasbourg.

Donnelan, K. (1966), "Reference and Definite Descriptions" The Philosophical Review 75:281-304.

von Fintel, K. (1994), Restrictions on Quantifier Domains, Ph.D. dissertation, Umass, Amherst.

Greenberg, Y. (1998), "Temporally Restricted Generics", in Proceedings of SALT 8, Cornell Linguistics Circle, Cornell University.

Greenberg, Y. (2002), "Manifestations of Genericity", Ph.D. dissertation, Bar-Ilan University

Jong, F. de and H. Verkuyl (1984) "Generalized Quantifiers: The Properness of their Strength" ,J. van Benthem and A, ter Meulen, eds., Generalized Quantifiers in natural Language, Foris, Dordecht.

Kadmon, N. and F. Landman (1993) "Any", Linguistics and Philosophy 16:353-422.

Kadmon, N (2001), Formal Pragmatics, Blackwell, Oxford.

Kratzer, A. (1981), "The Notional Category of Modality" in H. -J. Eikmeyer \& H. Reiser (eds.) Worlds and Contexts. New Approaches to World Semantics. de Gruyter, Berlin.

Krifka, M. (1987), An outline of Genericity Partly in collaboration with C. Gerstner, SNS -Bericht, University of Tubingen.

Krifka, M. (1995), "'Focus and the Interpretation of Generic Sentences" in G. Carlson and F.J. Pelletier (eds.) The Generic Book, The University of Chicago Press, Chicago.

Krifka M., Pelletier, F.J., Carlson G. ter Meulen A., Link, J. and G. Chierchia (1995). "Genericity: An Introduction" in G. Carlson and F.J. Pelletier (eds.) The Generic Book, The University of Chicago Press, Chicago.

Lawler, J. (1973) _Studies in English Generics University of Michigan Papers in Linguistics 1:1, University of Michigan Press, Ann Arbor.

Lewis, D. (1986) "Counterfactuals and Comparative Similarity" in D. Lewis Philosophical Papers. Oxford University Press.

Link, G. (1995) “Generic Information and Dependent Generics” in G. Carlson and F.J. Pelletier (eds.) The Generic Book, The University of Chicago Press, Chicago.

de Swart, H. (1994) "(In)definites and Genericity", ms. University of Groningen. ter Meulen, A., (1995) "Semantic Constraints on Type Shifting Anaphora" in G.

Carlson and F.J. Pelletier (eds.) The Generic Book, The University of Chicago Press, Chicago.

Wilkinson, C. (1991), "Studies in the Semantics of Generic Noun Phrases", Ph.D. dissertation, Umass, Amherst. 